\section{Wavefront aberration changes caused by a gradient of increasing accommodation stimuli}

X-Y Zhou ${ }^{1}$, L Wang ${ }^{1}$, X-T Zhou ${ }^{1,2}$ and Z-Q Yu ${ }^{1,2}$

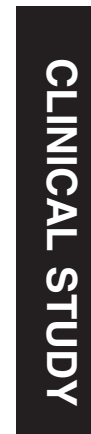

Eye (2015) 29, 115-121; doi:10.1038/eye.2014.244; published online 24 October 2014

Introduction

Accommodation is accomplished by changes in the shape of the crystalline lens. ${ }^{1}$ The central surface of the lens becomes steeper, whereas the periphery becomes flatter during accommodation. ${ }^{2}$ In addition, as optical power increases with accommodation, spherical aberration decreases. ${ }^{1,3,4}$

One theory that attempts to explain the phenomenon of these changes is the Helmholtz theory of accommodation. ${ }^{5}$ This theory asserts that the zonules are at maximal tension when the lens is at minimum optical power. In other words, when the eye is looking at a distant object, the equator of the lens moves toward the sclera, and then the ciliary muscle relaxes, increasing the zonule tension of the lens, and resulting in a flattened lens. When the eye is looking at a near object, the equator of the lens moves away from the sclera, and then the ciliary muscle contracts, the zonules of the lens relax, the lens becomes more convex owing to its elasticity, the optical power increases, the diameter of the lens reduces, and its anteroposterior diameter increases. However, the Helmholz theory does not account for the flattening of the peripheral surface of the lens, which occurs during accommodation. ${ }^{2}$

Furthermore, there is currently no working model of the human lens that can mimic its properties using the Helmholz principles. ${ }^{4}$

The Schachar theory of accommodation proposes that as the radial fibers of the ciliary muscle contract the equatorial zonule tension increases, and that as the circular fibers of the
${ }^{1}$ Department of Ophthalmology, Eye \& ENT Hospital, Fudan University, Shanghai, China

${ }^{2}$ Key Laboratory of Myopia, Ministry of Health, Shanghai, China

Correspondence: Z-Q Yu, Department of Ophthalmology, Eye \& ENT Hospital, Fudan University, 19 Baoqing Road, Shanghai 200031, China Tel: +86 2164377134 ; Fax: +86 2164318258 . E-mail: zhqyu@21cn.com

Received: 28 February 2014 Accepted in revised form: 2 September 2014 Published online: 24 October 2014 
ciliary muscle contract the anterior and posterior zonules relax. As the tension on the equator of the lens increases, its diameter increases. Thus, the central portion of the lens becomes more convex (increases in volume), and the peripheral portion flattens (decreases in volume). As the surface curvature of the central portion of the lens increases, the central optical power increases. In addition, as the peripheral portion flattens, the optical power decreases, and the spherical aberration is negative. A working model of the human crystalline lens, based on Schachar's theory of accommodation, has been produced. ${ }^{6}$ This model can demonstrate changes in optical power of $>10$ diopters (D), with concomitant reductions in spherical aberration.

Studies involving the correlation between changes in spherical aberration with changes in accomodation have provided variable results. ${ }^{7-11}$ Moreover, the magnitude and direction of the change in coma have been inconclusive. Each refractive interface (ie, pupil size, corneal shape, lens shape, and retinal topography) can affect wavefront aberration. Few studies have evaluated wavefront aberration in dilated eyes in young adults over different levels of accommodation. This approach theoretically should avoid interference due to pupil contraction and aging, and thus all changes in optical aberrations can be attributed to changes in the contour of the crystalline lens.

The gradient of increasing accommodation stimuli is not a routine examination, and it was designed for this study. The purpose of this study was to investigate the wavefront aberration changes in human eyes caused by a gradient of accommodation stimuli (0-6D). The results of the current study support the Schachar theory of accommodation.

\section{Materials and methods}

\section{Study participants}

Twenty-two right eyes of 22 healthy volunteers from Fudan University were enrolled in this study. The study was conducted in accordance with the tenets of the Declaration of Helsinki and was approved by the Institutional Review Board of the university. All participants provided informed consent before participation in the study. Refraction status among these volunteers ranged from 0 to $-1 \mathrm{D}$, with astigmatism $<1 \mathrm{D}$. Therefore, the low-order aberrations were standardized, thus highlighting the changes in the highorder aberrations. As the maximum amount of the accommodation stimuli was $6 \mathrm{D}$ in the study, it was required that the amplitude of accommodation of the included volunteers was $>6 \mathrm{D}$ so that it could be ensured that the maximum gradient of accommodation stimuli could be given. We initially planned to use emmetropic volunteers. However, in the process of selecting the study participants, students rarely achieved complete emmetropia. In China, incidence of myopia is $>85 \%$, and the remaining $15 \%$ of students are mostly associated with hyperopia or astigmatism. Therefore, volunteers with mild myopia were also selected.

\section{Apparatus}

The WFA-1000B aberrometer (Bright Eye Medical Device Co, Ltd, Suzhou Industrial Park, Suzhou, Jiangsu Province, China) was used for the measurement of aberrations. The instrument can depict aberrations of the eye using a wave aberration map and Zernike aberration function. The aberrometer can measure 35 Zernike aberrations and diopter simultaneously, and it can continuously increase the built-in negative spherical lens in front of the eye, providing a gradient of accommodation stimuli. Its separated aberration function can measure 35 aberrations, including the defocus measured by conventional optometers (myopia or hyperopia) and astigmatism. Diopter, wavefront aberration, and Zernike aberration measurements were performed. The minimum pupil diameter that could be measured was $\sim 4.5 \mathrm{~mm}$. The instrument was equipped with built-in fixation capabilities. The PD measurement was set at $80 \mathrm{~mm}$. The scopes of movement provided by the instrument body were as follows: $77 \mathrm{~mm}$ anteroposteriorly, $30 \mathrm{~mm}$ vertically, and $90 \mathrm{~mm}$ laterally. The jaw holder allowed a scope of movement of $75 \mathrm{~mm}$.

The objective type of aberrometer selected was based on the Hartmann-Shack principle. The instrument tracks the optics light path to measure aberrations. The built-in negative spherical lens may introduce some spherical aberration. A subjective type of aberration instrument was used in this study. The Zernike coefficients were calculated according to the examinee's reactions after observing the visual targets. The built-in negative spherical lens can cause defocus effects. Examinees have experienced stimuli of defocus effects and automatically offset the defocus impact to regain clear vision.

Measurements with a subjective type aberrations instrument will generally not be affected by built-in negative spherical lens' spherical aberration.

\section{Examination conditions}

Examinations were carried out in a dark room under the conditions of $9.5 \mathrm{~mm}$ dilated pupil after the $0.5 \%$ phenylephrine drops were administered. Patients were asked to sit in front of the aberrometer. The patients were asked to place their jaws on the jaw holder and gaze straight ahead. The patients were instructed to maintain a stable head position during the examination. 
Pupil diameters were measured with a NIDEK (Gamagori, Japan) ARK-700A Autorefractor.

\section{The measurement process}

According to each corresponding condition of the patients, including pupil size, diopter, and degree of astigmatism, the physician will choose whether or not to use the automatic tracking system. The automatic tracking system provided big pupil or small pupil for measurement, the compensation lens (CR), and the astigmatism auxiliary examination function (CY). When a patient's degree of astigmatism was $>2 \mathrm{D}$, the astigmatism auxiliary examination function was enabled (CY).

\section{Zernike polynomials}

Zernike polynomials can represent a wavefront aberrations (wavefront aberration) function and the characteristic function of the orthogonal wave measurements. This method of computation decomposes the irregular surface of the cornea into a series of simpler mathematical components. ${ }^{12}$ The accuracy of Zernike polynomials in fitting wavefront aberration depends on the number of terms applied. ${ }^{13}$ The more the terms used, the closer the root mean square (RMS) value is to 0 $(0=$ a perfect fit). The Zernike coefficients obtained for the current study represent all 35 seventh-order aberrations.

\section{Statistical analysis}

The RMS values of the total wavefront aberrations, higher-order aberrations, and 35 individual Zernike aberrations under different accommodation stimuli were calculated and presented (mean and standard deviation (SD)). The significance of the difference between relaxed accommodation and each accommodation stimuli was determined by paired $t$-test. Repeated measures analysis of variance was used to compare the seven accommodation stimuli (relaxed, 1-6D) among subjects, where applicable. For repeated measures, sphericity was confirmed using the Mauchly's sphericity test. The Greenhouse-Greiser correction for sphericity was applied if the assumption of sphericity had been violated. All statistical assessments were two-sided and evaluated at the 0.05 level of significance. Statistical analyses were performed using the SPSS 18.0 statistics software (SPSS Inc, Chicago, IL, USA).

\section{Results}

A total of 10 men and 12 women were enrolled in this study. The average age is $22.0 \pm 2.8$ years (ranging from
18 to 28 years). The average Snellen chart is $20 / 25$ (ranging from $20 / 15$ to $20 / 40$ ). The average sphere is -0.3 (ranging from -0.75 to 0 ). The average astigmatism is -0.3 (ranging from -0.75 to 0 ). The average induced accommodations with 1, 2, 3, 4, 5, and $6 \mathrm{D}$ accommodation stimuli were $0.748 \mathrm{D}, 1.526 \mathrm{D}$, $2.375 \mathrm{D}, 3.249 \mathrm{D}, 4.181 \mathrm{D}$, and $5.085 \mathrm{D}$, respectively (Figure 1). The mydriatic used in this study had no cycloplegic effect, and it should not cause drug-induced lag. However, normal adjustment lag existed. From Figure 1, one can clearly observe adjustment lag (accommodation stimulus subtracted induced accommodation) and an eye could not achieve a full $6 \mathrm{D}$ accommodation after a 6D stimulus.

The RMS of total wavefront aberrations, as well as higher-order aberrations at 1-3 D accommodation stimuli, was not significantly different from that obtained with relaxed accommodation, but the RMS values of total wavefront aberrations and higher-order aberrations at 4-6 D accommodation stimuli were significantly higher than that achieved with relaxed accommodation; all $P$-values $\leq 0.030$ Figure 2 a vs all $P$-values $<0.010$, Figure $2 b$, respectively. Furthermore, the changes in the RMS values of total wavefront aberrations and higherorder aberrations were significantly different among different accommodation stimuli $(P$-value $=0.006$ and $P$-value $<0.001$, respectively).

In second-order Zernike polynomials, the Zernike coefficient of $Z_{2}^{0}$ at all 1-6 D accommodation stimuli was significantly lower than that with relaxed accommodation (all $P$-values $<0.010$, Figure 3 ). The change of $Z_{2}^{0}$ was significantly different among the various accommodation stimuli $(P$-value $<0.001)$. The Zernike coefficients of $Z_{2}^{-2}$ and $Z_{2}^{2}$ at all $1-6 \mathrm{D}$ accommodation stimuli were not significantly different compared with that with relaxed accommodation.

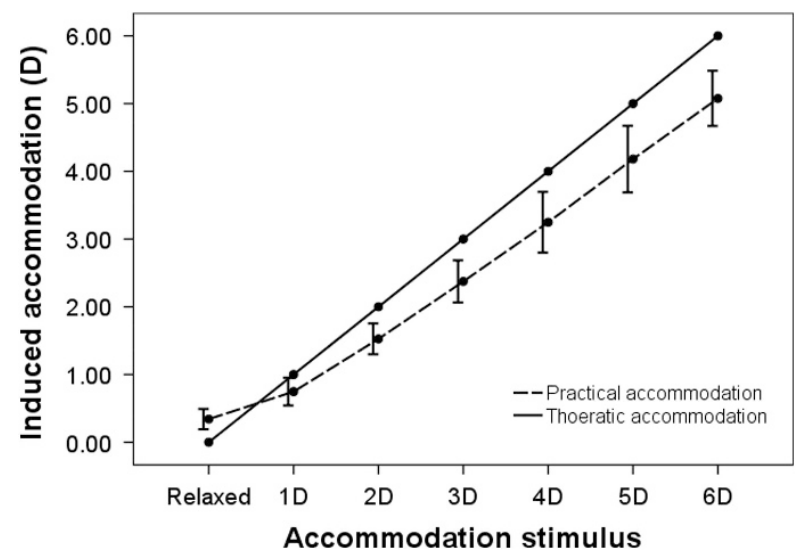

Figure 1 The average induced accommodation with 1-6D accommodation stimulus. 
a

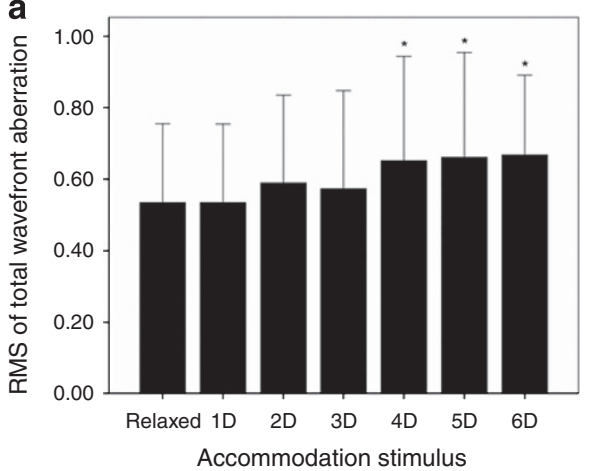

b

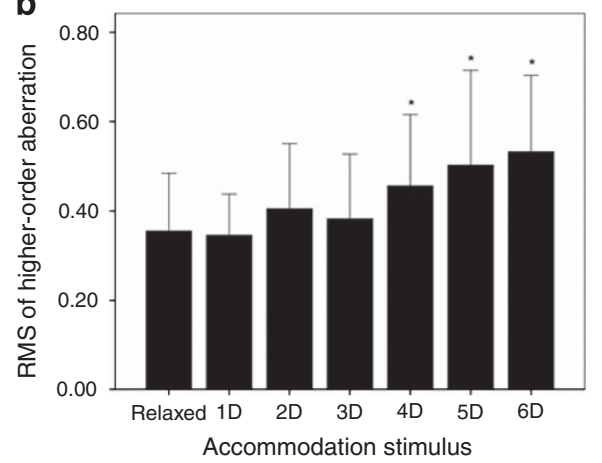

Figure 2 (a) Average change in each accommodation stimulus of total wavefront aberration. Bars represent mean \pm SD. *Indicates significant difference compared with relaxed accommodation. (b) Average change in each accommodation stimulus of high-order aberration. Bars represent mean \pm SD. *Indicates significant difference compared with relaxed accommodation.

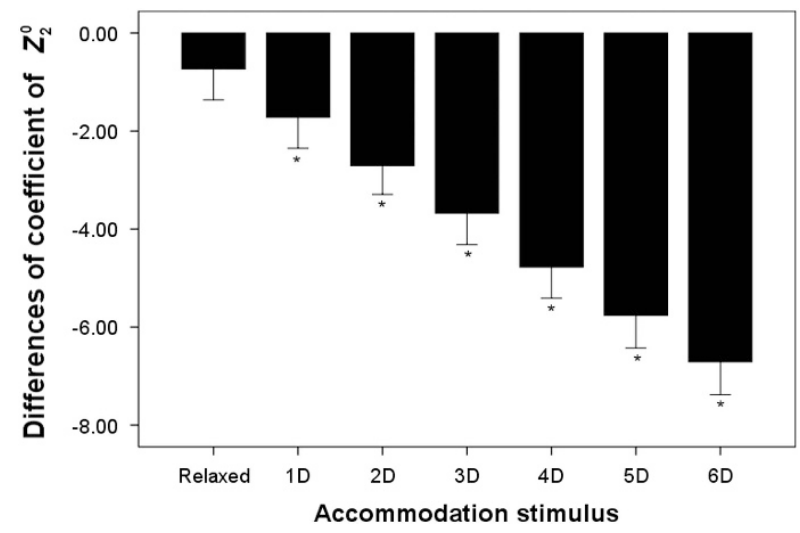

Figure 3 Average change in each accommodation stimulus of $Z_{2}^{0}$. Bars represent mean $\pm S D$. ${ }^{*}$ Indicates significant difference compared with relaxed accommodation.

In third-order Zernike polynomials, the Zernike coefficients of $Z_{3}^{-3}, Z_{3}^{-1}, Z_{3}^{1}$, and $Z_{3}^{3}$ at all $1-6 \mathrm{D}$ accommodation stimuli were not significantly different compared with that with relaxed accommodation.

In fourth-order Zernike polynomials, the Zernike coefficients of $Z_{4}^{0}$ at the 2-6D accommodation stimuli were significantly lower than that with relaxed accommodation (all $P$-values $<0.020$, Figure $4 a$ ). The change of $Z_{4}^{0}$ was significantly different among different accommodation stimuli $(P$-value $<0.001)$. The Zernike coefficient of $Z_{4}^{2}$ only at 3,4 , and $6 \mathrm{D}$ accommodation stimuli were significantly higher than that with relaxed accommodation (all $P$-values $<0.020$, Figure $4 b$ ). The change of $Z_{4}^{2}$ was significantly different among the various accommodation stimuli $(P$-value $=0.005)$. The Zernike coefficients of $Z_{4}^{-4}, Z_{4}^{-2}$, and $Z_{4}^{4}$ at all $1-6 \mathrm{D}$ accommodation stimuli were not significantly different compared with that with relaxed accommodation.

In fifth-order Zernike polynomials, the Zernike coefficient of $Z_{5}^{-5}$ only at the $3 \mathrm{D}$ accommodation
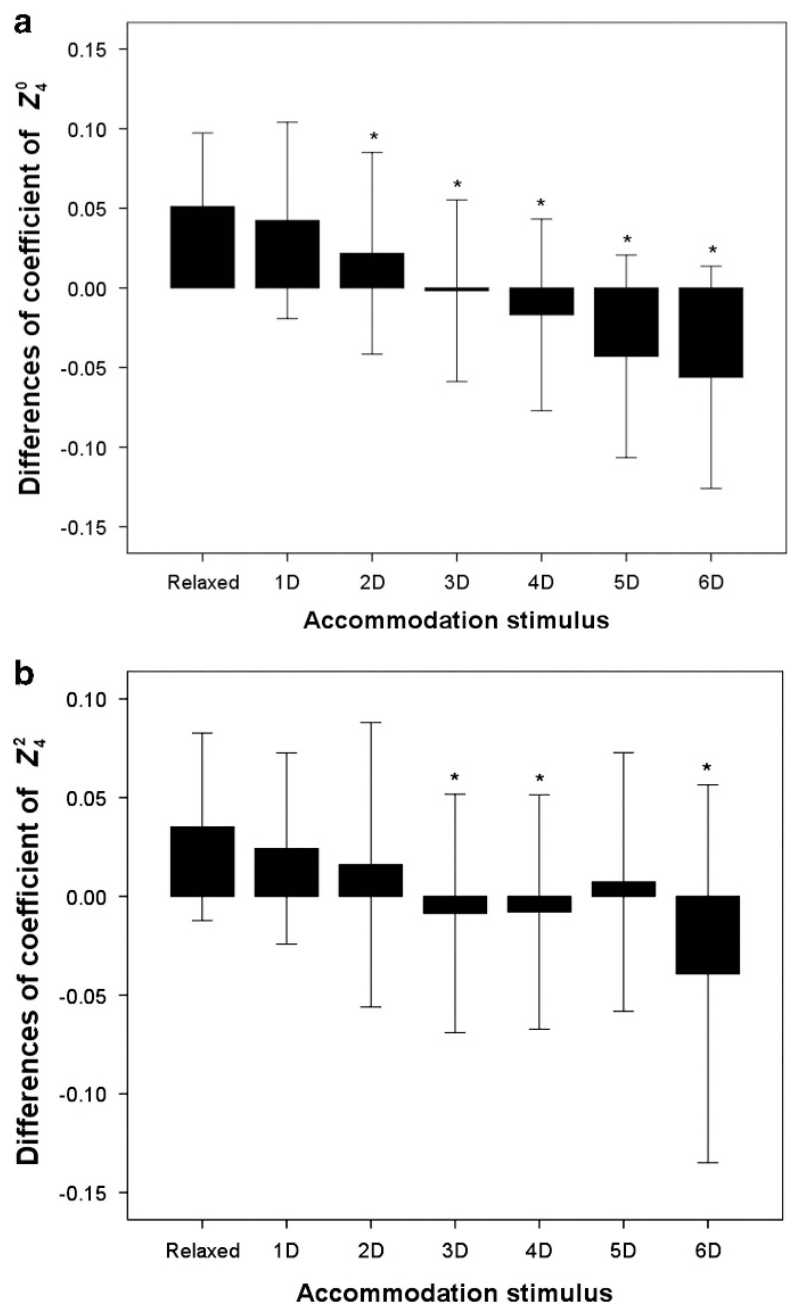

Figure 4 (a) Average changes in each accommodation stimulus of $Z_{4}^{0}$. Bars represent mean $\pm S D$. (b) Average change in each accommodation stimulus of $Z_{4}^{2}$. Bars represent mean $\pm S D$. *Indicates significant difference compared with relaxed accommodation. 
stimulus was significantly higher than that with relaxed accommodation ( $P$-value $=0.044$, data not shown). The Zernike coefficient of $Z_{5}^{-3}$ was significantly higher than that with relaxed accommodation only at the $3 \mathrm{D}$ accommodation stimulus ( $P$-value $=0.042$, data not shown). The Zernike coefficient of $Z_{5}^{-1}$ was significantly higher than that with relaxed accommodation only at the $3 \mathrm{D}$ accommodation stimulus $(P$-value $=0.018$, data not shown). The Zernike coefficients of $Z_{5}^{1}, Z_{5}^{3}$, and $Z_{5}^{5}$ at all 1-6D accommodation stimuli were not significantly different compared with that with relaxed accommodation.

In sixth-order Zernike polynomials, the Zernike coefficients of $Z_{6}^{0}$ at 3-6D accommodation stimuli were significantly lower than that with relaxed accommodation (all $P$-values $<0.050$, data not shown). The change of $Z_{6}^{0}$ was significantly different among different accommodation stimuli $(P$-value $=0.005)$. The Zernike coefficients of $Z_{6}^{-6}, Z_{6}^{-4}, Z_{6}^{-2}, Z_{6}^{2}, Z_{6}^{4}$, and $Z_{6}^{6}$ at all $1-6 \mathrm{D}$ accommodation stimuli were not significantly different compared with that with relaxed accommodation.

In seventh-order Zernike polynomials, the Zernike coefficients of $Z_{7}^{-7}$ at 4-6D accommodation stimuli were significantly higher than that achieved with relaxed accommodation ( $P$-value $<0.020$, data not shown). The change of $Z_{7}^{-7}$ was significantly different among the various accommodation stimuli $(P$-value $=0.045)$. The Zernike coefficient of $Z_{7}^{-5}$ was significantly higher than that with relaxed accommodation only at the $3 \mathrm{D}$ accommodation stimulus ( $P$-value $=0.017$, data not shown). The Zernike coefficient of $Z_{7}^{-3}$ was significantly higher than that with relaxed accommodation at the 3and $6 \mathrm{D}$ accommodation stimuli ( $P$-value $<0.040$, data not shown). The Zernike coefficient of $Z_{7}^{3}$ was significantly higher than that with relaxed accommodation only at the $6 \mathrm{D}$ accommodation stimulus $(P$-value $=0.012$, data not shown). The Zernike coefficient of $Z_{7}^{5}$ was significantly higher than that with relaxed accommodation only at the $6 \mathrm{D}$ accommodation stimulus $(P$-value $=0.033$, data not shown). The Zernike coefficients of $Z_{7}^{-1}, Z_{7}^{1}$, and $Z_{7}^{7}$ at all 1-6D accommodation stimuli were not significantly different compared with that with relaxed accommodation.

After full analysis of all 35 categories, except for aforementioned results, no statistically significant differences were found among all other subcategories.

\section{Discussion}

In the current study of 22 patients, RMS values for total wavefront aberration and higher-order aberration both showed significant effects at the higher levels of accommodation (4-6D). In general, the Zernike coefficients of $Z_{4}^{0}, Z_{2}^{0}, Z_{4}^{2}$, and $Z_{7}^{-7}$ became increasingly negative (compared with relaxed accommodation) with increased levels of accommodative stimuli up to $6 \mathrm{D}$. Conversely, $Z_{6}^{0}$ significantly increased with increasing levels of accommodation. The spherical aberration $Z_{4}^{0}$ and the secondary spherical aberration $Z_{6}^{0}$ are both closely related to the curvature changes of the central and the peripheral portions of the lens. When accommodation occur, a change of curvature of the periphery lens happens; so the spherical aberration and the secondary spherical aberration corresponding changes have taken place.

Although some data points, including Zernike coefficients of $Z_{5}^{-5}, Z_{5}^{-3}, Z_{5}^{-1}, Z_{7}^{-5}, Z_{7}^{-3}, Z_{7}^{3}$, and $Z_{7}^{5}$, were significantly different from relaxed accommodation, they did not exhibit clear trends with increasing levels of accommodation stimuli. Wavefront errors in human eyes exhibit complex irregular shapes, which vary considerably between individuals. ${ }^{7,14}$ Spherical aberration, coma aberrations, other asymmetric aberrations, and those with orders higher than the fifth order can contribute a large degree to reducing visual performance when the pupil is dilated.9,15-18 Aberrations in the human eye can change between individuals in both their amplitude and sign. ${ }^{14,16-19}$

We did not observe positive results for primary astigmatism (vertical $Z_{2}^{-2}$ or oblique $Z_{2}^{2}$ ), primary coma (vertical $Z_{3}^{-1}$ or horizontal $Z_{3}^{1}$ ), or low-order trefoil (vertical $Z_{3}^{-3}$ or oblique $Z_{3}^{3}$ ). This may have been due to individual variations in response in our particular patient cohort. Atchison et $a l^{9}$ also could not find a clear trend of variance in the wave aberrations with changes in accommodation. Only about half of their patients exhibited a trend toward a negative spherical aberration with accommodation. He et $a l^{7}$ pointed out that a change in coma displays a more complex pattern of individual variation that causes a change in spherical aberration.

The RMS value, which is currently the most common metric used to describe wavefront aberration, gives an estimation of the variation in the wavefront error of a pupil size of interest. ${ }^{20}$ In the present study, lower levels of accommodation had little effect on average, but stimuli at or above $4 \mathrm{D}$ produced increases in the RMS values of both total and higher-order wavefront aberration. These results are consistent with those of $\mathrm{He}$ et $a l^{7}$ in their study of eight patients, who found slight decreases in RMS values between 0 and $-1 \mathrm{D}$, no change between -1 and $-3 \mathrm{D}$, and gradual increases from $-4 \mathrm{D}$ to $-6 \mathrm{D}$ of accommodation. In agreement with the current study, Atchison et $a l^{9}$ observed no clear trend in the variation of wavefront aberration with accommodative stimuli of $0-3 \mathrm{D}$. Their stimuli may have been inadequate to produce a clear trend. Another study of 91 optometry students found increases in RMS 
aberration with increasing accommodation for Zernike coefficients Z3 and Z5 combined (astigmatism, slope $=0.0134), Z 7$ and Z8 combined (coma, slope $=0.0135$ ), and Z12 (spherical aberration, slope $=0.0428) .{ }^{10}$ In that study, the rates of change of astigmatism and coma were about $1 / 3$ that of the spherical aberration with a $5-\mathrm{mm}$ pupil. Another study of 60 participants confirmed that the higher-order RMS values increased with increasing accommodation. ${ }^{21}$ Interestingly, when the data were analyzed according to age groups, the slope of lines through those data points were greater with age (ie, 50- to 60-year-old group). He et $a l^{7}$ concluded that higher-order aberrations are correlated with the overall accommodative effort and have a major effect on the decreased image quality observed with marked accommodation. All the aforementioned study results corroborate those of the current study, which indicate larger values for total wavefront aberration and higher-order aberration with large accommodation stimuli $\geq 4 \mathrm{D}$.

Ocular aberrations are known to change as the eye accommodates to focus on objects at different distances. ${ }^{7}$ These aberrations occur owing to the optical quality of the ocular tissues that transmit light and from misalignment in position of different components of the visual system. In the present study, the pupil diameters were kept consistent in size in order to minimize bias in the subsequent measurements. The accommodation stimuli were generally successful at producing induced levels of accommodation that were close to the expected theoretic levels. Increasing levels of accommodation caused the average Zernike coefficients of spherical aberration to become increasingly negative. A similar trend was reported by Hazel et al in their study with 30 healthy participants (10 emmetropes and 20 myopes) between 18 and 27 years of age. ${ }^{22}$ Cheng et al found that spherical aberration was the most affected of the Zernike terms evaluated, with the negative change in spherical aberration being proportional to the accommodative response. ${ }^{10}$ Others have reported that decreases in the spherical aberration increased the accommodation stimuli. ${ }^{7,23}$ Thibos et al hypothesize that hyperopic blur can be a risk factor for the progression of myopia when the eye has a negative spherical aberration (which can lead to low contrast in a defocused retinal image). ${ }^{24,25}$

Changes in ocular wavefront aberrations occur as accommodation attempts to compensate for retinal defocus. ${ }^{11}$ The average change in defocus in the present study showed a clear negative relationship with increasing accommodation stimuli. Hampson et $\mathrm{al}^{26}$ have used adaptive optics to study the effects of ocular aberration on the chaos in the accommodative control system. They found reductions in the chaotic nature of accommodative fluctuations during the process of defocus correction. Fluctuations in defocus had a more pronounced effect in terms of stress to the ocular system than did other ocular aberrations.

A recent study by Yuan et al ${ }^{11}$ evaluated the correlations between anterior segment biometry and high-order aberrations during accommodation. They found that anterior chamber depth, pupil diameter, and radii of the lens surface decreased, whereas lens thickness and the RMS of high-order aberration (fixed 3-mm pupil size) increased with accommodative stimuli. In general, anterior segment biometry and high-order aberration showed significant changes during accommodation. The authors proposed that the increases in high-order aberrations were mainly caused by increases in the convexity of the anterior surface of the crystalline lens during the process of accommodation. ${ }^{11}$

The current study was relatively small, and therefore caution must be used when generalizing these results to the general population. Several studies have indicated that high levels of individual variation exist in terms of the effects of accommodation on wavefront aberrations. $7,9,22,27$ Owing to the large number of the types of aberrations evaluated in the present study, only mean results are reported herein. A large-scale study, of similar design, including participants of different ages and ethnicities would provide additional data to more fully characterize the wavefront aberrations that take place during accommodation.

Higher-order wavefront aberrations in human eyes changed with increasing levels of accommodation. The observed changes of spherical aberration are related to surface curvature changes of the crystalline lens and the cornea. When the pupils were dilated to a diameter of $9.5 \mathrm{~mm}$, our results showed that changes to the shape of the peripheral portion of the lens also caused changes in aberrations in the peripheral region of the lens. When accommodation occurs, the crystalline lens periphery is flattened, causing greater negative spherical aberration. The results of this study are consistent with Schachar's theory of accommodation.

\section{Summary}

What was known before

- Higher-order wavefront aberrations changed with increasing levels of accommodation.

- Helmholz theory does not account for the flattening of the lens during accommodation.

What this study adds

- To evaluate wavefront aberration in young adults over different accommodations.

- Dilated 9.5-mm pupils will cause changes in aberrations of lens' peripheral region. 


\section{Conflict of interest}

The authors declare no conflict of interest.

\section{References}

1 Young T. On the mechanism of the eye. Phil Trans Roy Soc 1801; 92: 23-88.

2 Fincham EF. Mechanism of accommodation. Br J Ophthalmol 1937; 92(Suppl): 5-80.

3 Koomen M, Tousey R, Scolnik R. The spherical aberration of the eye. J Opt Soc Am 1949; 39: 370-376.

4 Schachar RA, Bax AJ. The mechanism of human accommodation as analyzed by nonlinear finite element analysis. Compr Ther 2001; 27: 122-132.

5 von Helmholtz H. Uber die akkommodation des auges. Albrecht von Graefe's Arch Ophthalmol 1855; 1: 1-89.

6 Schachar RA, Cudmore DP, Black TD. A revolutionary variable focus lens. Ann Ophthalmol 1996; 28: 11-18.

7 He JC, Burns SA, Marcos S. Monochromatic aberrations in the accommodated human eye. Vision Res 2000; 40: 41-48.

8 Ninomiya S, Fujikado T, Kuroda T, Maeda N, Tano Y, Oshika T et al. Changes of ocular aberration with accommodation. Am J Ophthalmol 2002; 134: 924-926.

9 Atchison DA, Collins MJ, Wildsoet CF, Christensen J, Waterworth MD. Measurement of monochromatic ocular aberrations of human eyes as a function of accommodation by the Howland aberroscope technique. Vision Res 1995; 35: 313-323.

10 Cheng H, Barnett JK, Vilupuru AS, Marsack JD, Kasthurirangan S, Applegate RA et al. A population study on changes in wave aberrations with accommodation. J Vis 2004; 4: 272-280.

11 Yuan Y, Shao Y, Tao A, Shen M, Wang J, Shi G et al. Ocular anterior segment biometry and high-order wavefront aberrations during accommodation. Invest Ophthalmol Vis Sci 2013; 54: 7028-7037.

12 Smolek MK. Method for expressing clinical and statistical significance of ocular and corneal wave front error aberrations. Cornea 2012; 31: 212-221.

13 Smolek MK, Klyce SD. Goodness-of-prediction of Zernike polynomial fitting to corneal surfaces. J Cataract Refract Surg 2005; 31: 2350-2355.

14 Liang J, Grimm B, Goelz S, Bille JF. Objective measurement of wave aberrations of the human eye with the use of a Hartmann-Shack wave-front sensor. J Opt Soc Am A Opt Image Sci Vis 1994; 11: 1949-1957.

15 Campbell MC, Harrison EM, Simonet P. Psychophysical measurement of the blur on the retina due to optical aberrations of the eye. Vision Res 1990; 30: 1587-1602.

16 Howland HC, Howland B. A subjective method for the measurement of monochromatic aberrations of the eye. J Opt Soc Am 1977; 67: 1508-1518.

17 Liang J, Williams DR. Aberrations and retinal image quality of the normal human eye. J Opt Soc Am A Opt Image Sci Vis 1997; 14: 2873-2883.

18 Walsh G, Charman WN, Howland HC. Objective technique for the determination of monochromatic aberrations of the human eye. J Opt Soc Am A 1984; 1: 987-992.

19 He JC, Marcos S, Webb RH, Burns SA. Measurement of the wave-front aberration of the eye by a fast psychophysical procedure. J Opt Soc Am A Opt Image Sci Vis 1998; 15: 2449-2456.

20 Pepose JS, Applegate RA. Making sense out of wavefront sensing. Am J Ophthalmol 2005; 139: 335-343.

21 Lopez-Gil N, Fernandez-Sanchez V, Legras R, Montes-Mico R, Lara F, Nguyen-Khoa JL. Accommodationrelated changes in monochromatic aberrations of the human eye as a function of age. Invest Ophthalmol Vis Sci 2008; 49: 1736-1743.

22 Hazel CA, Cox MJ, Strang NC. Wavefront aberration and its relationship to the accommodative stimulus-response function in myopic subjects. Optom Vis Sci 2003; 80: 151-158.

23 Fritzsch M, Dawczynski J, Jurkutat S, Vollandt R, Strobel J. [Monochromatic aberration in accommodation. Dynamic wavefront analysis]. Ophthalmologe 2011; 108: 553-560.

24 Thibos LN, Bradley A, Liu T, Lopez-Gil N. Spherical aberration and the sign of defocus. Optom Vis Sci 2013; 90: 1284-1291.

25 Gwiazda JE, Hyman L, Norton TT, Hussein ME, Marsh-Tootle W, Manny R et al. Accommodation and related risk factors associated with myopia progression and their interaction with treatment in COMET children. Invest Ophthalmol Vis Sci 2004; 45: 2143-2151.

26 Hampson KM, Cufflin MP, Mallen EA. Effect of correction of aberration dynamics on chaos in human ocular accommodation. Opt Lett 2013; 38: 4747-4749.

27 Collins MJ, Wildsoet CF, Atchison DA. Monochromatic aberrations and myopia. Vision Res 1995; 35: 1157-1163. 